\title{
IUE ULTRAVIOLET SPECTRA OF V SAGITTAE
}

\author{
Robert H. Koch \\ and \\ M. Javad Siah \\ and \\ Michael N. Fane111 \\ Department of Astronomy and Astrophysics \\ University of Pennsylvanta
}

$V$ Sge is a short period, double-line spectroscopic and eclipsing close binary which is also intrinsically variable through a range of at most 3 mag in visual and photographic light. Almost all modern insight is founded upon the interpretation by Herbig, et al. (1965), who developed the evidence for the binary nature of the star, evaluated the nearly 0.5 day Keplerian perlod, studied the short-term Doppler and intensity variations of the spectrum, and obtained a limiting representation of the intrinsically noisy ultraviolet light curve. The orbital plane view of the binary, as developed by these authors, shows patchy distributions of 0 IIIfl and $0 . V I$ emfssions and mass loss from the system as a whole.

In view of the facts that (a) the Herbig, et al. study predates modern modeliing of cataclysmic binaries as summarized, e.g., by Robinson (1976) and (b) numerous cataclysmic variables have periods up to several thousand days, it seemed useful to re-examine the evidence for cyclical eruptions for V Sge. The light curves by Campbell (1912, 1918, 1938), Jacchia (1931), Ryves (1932), the timings of maximum light by Gaposchkin (1952), and numerous remarks in the recent J.R.A.S. Canada attributed to AAVSO observers have all been scrutinized anew. There are obvious seasonal selection effects in these estimates and measures and, when these effects are removed, an outburst cycle length between 1.2 and 1.8 years is very strongly suggested over the interval from 1904 to 1976. Such an outburst cycle length is characteristic of dwarf novae. Superposed upon this variabllity are much higher frequency outbursts and declines and these in turn are inflected by the Keplerian eclipse modulation.

Unt1l recently, it has been difficult to determine if the Keplerian period is constant. Partly, this is due to the intrinsic variability of the minima; even photoelectric determinations show noise which is surprisingly large. By now, however, almost 21000 cycles of photometric 
history exist and all of these minima, faced against the ephemeris by Berbig, et al., lead to an improved ephemeris:

Hel. PI. MIn. $=2437889.9136+0.5142180 \mathrm{E}-\left(0.86 \times 10^{-10}\right) \mathrm{E}^{2}$. (1)

This fit to the residuals is significantly better than is elther IInear improvements to the period and epoch or a cublc in $E$. There can be no doubt that the period has been decreasing. If the period diminution be associated only with the systemic mass loss seep spectrographically and If the mass loss were 1sotropic, $\Delta m=8.2 \times 10^{-10} \mathrm{~m}$, where $m$ is counted In units of the solar mass. For a systemic mass $\approx 3 \odot$, the mass loss would be of the order of $9 \times 10^{-8} \odot / y r$.

In $1978 \mathrm{~V}$ Sge was observed on the IUE program PG2SS, ortginally concelved by S. Sobleski. The star is so fatnt and the Keplerlan perlod so short that only low dispersion spectra $(R \simeq 6 \&$ ) could be obtained. In all, 9 SWP (1150 $<<<1950 \AA)$ and 9 LWR (1850 \&< $\lambda<$ 3300 \&) images were obtalned, but two spectra are unusually noisy and three of the LWR spectra are saturated through part of the dispersion. Exposure times ranged from 9 to 40 minutes. The spectra are equally divided between large and small aperture exposures, and conversion of the fluxes from small to large aperture data is based upon the assumption that no significant changes occur over Intervals as short as 10 and as long as 36 minutes. The maxima of the eclipsing light curve are sampled by 8 spectra, secondary minimum by 2 spectra, and primary minimm by only 8 spectra.

Representative spectra are shown in Fig. 1 and 2. As can be

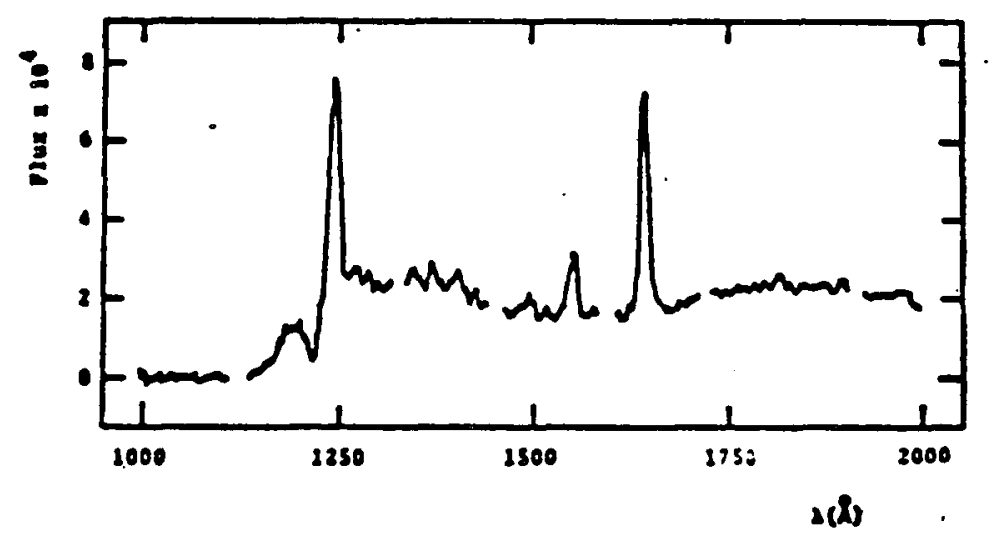

F1g. 1 - Background-subtracted, natural system spectrm of V Sge, $1150 \AA$ $<\lambda<1950$ \&.

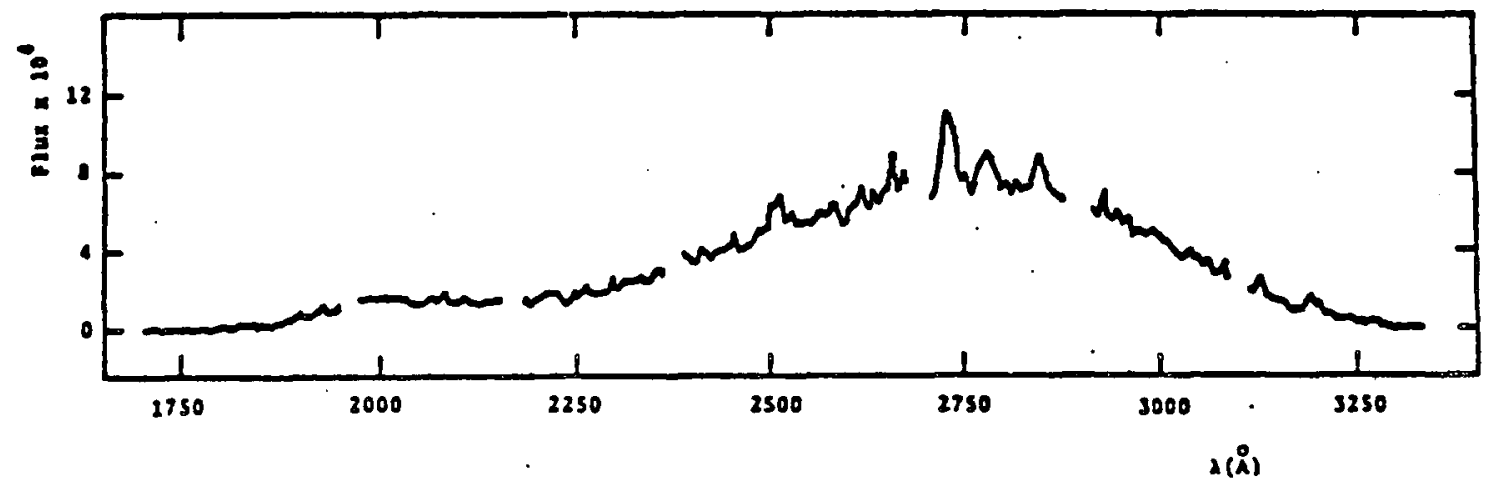

Fig. 2 - Background-subtracted, natural system spectrum of V Sge, $1850 \AA$ $<\lambda<33008$. 
seen, the appearance of the spectra is dominated by emission features. The only absorption which can be detected is $L \alpha$ and 1 ts profile is sometimes cut up by geocoronal emission, the strength of which depends upon the exposure time and chosen aperture. The spectral resolution is so low that the observed wavelength of the $L \alpha$ absorption cannot be tested against the expansion velocitles observed by Herbig, et al. for the weak Balmer absorption reversals. As nearly as can be measured, the equivalent width of the La absorption is $\simeq 8 \AA$, but this is 1ikely a lower limit since stellar He II $\lambda 1215$ and $L \alpha$ could also be in emission for this star.

As nearly as possible, a smooth free-hand continuum was drawn on each spectrum taking account of the noise level and the emission features. At intervals of $50 \AA$ these smoothed continuum levels were corrected for the cathode responses and the famlliar interstellar absorption dip near $2200 \&$ was evident. The reddening law given by Jamar, et al. (1976) was assumed and sample values of $E(B-V)$ were tested in order to remove the interstellar $d i p$ and to avold a spurious bump. A value of $E(B-\nabla)=0.20$ was found. This may be compared to the value of 0.40 , associated with a distance of $2.75 \mathrm{kpc}$, chosen by Herbig, et al. to be the best compromise from determinations by UBV photometry, the absence of galaxies in this field, and the strength of the interstellar K-line. Correction for a value as large as 0.40 introduces a bump into the ultraviolet spectrum. On the other hand, Fitzgerald (1968) finds $0.0<E(B-V)<0.1$ out to a distance of $1.5 \mathrm{kpc}$ in the field of $\mathrm{V}$ Sge. A value of $E(B-V)$ this low, however, leaves an absorption dip near 22200. A distance of $\approx 2 \mathrm{kpc}$ is suggested as the best determination consistent with the IUE spectra.

The spectra were obtained over an interval of 179 days. The FES monitor on board the spacecraft provides $\underline{V}$ magnitudes (to $\pm 0.1 \mathrm{mag}$ ), and these were compiled in phase by ephemeris (1). These $\underline{V}$ observations do not yleld a phase-locked eclipsing light curve of the kind obtained by Herbig, et al., and this must be due, in part, to the intrinsic variability over such a long perfod of time. Fluxes at 6 wavelengths ( 3 each from the SWP and LWR spectra) apparently free of line emission have been phased by ephemeris (1). These also do not show the famfliar eclipsing-type light curve although phase coverage is far from dense. It is clear, therefore, that the intrinsic variability, known from ground-based observing, also occurs in the satellite UV interval.

The calibrated, reddening-corrected fluxes were then studied in order to derive a temperature. This entalled plecing together SWP and LWR spectra obtained over an interval as long as $0.08=0.16 \mathrm{P}$. If variability has occurred over time scales shorter than this interval, it will have been ignored in the plecing together of the SWP and LWR spectra. Several possibilities were investigated. (1) The masses and radil given by Herbig, et al. could suggest non-degenerate stars. Therefore, H-rich atmospheres from Carbon and Gingerich (1969) were tested against the IUE continuum fluxes. For temperatures as low as are suggested by the masses and radil, no f1t whatever could be obtalned to the slope of the IUE fluxes. (2) For H-rich model atmospheres hotter than the masses and radil would suggest, fallure was 
also conspicuous. (3) Considerably greater success was attained with white dwarf model atmospheres privately provided by H. L. Shipman. For $\lambda>1250$, spectra SWP 2218 and 2234 and LWR 1993 and 2012, obtained wthin an interval of 2 days, were adequately represented by: $\mathrm{T}_{\mathrm{e}}=17000 \mathrm{~K}, \log \mathrm{g}=8, \mathrm{n}(\mathrm{He})=1$, solar $\mathrm{n}$ (metals). There is, however, somewhat too much stellar flux for $\lambda<1200$. (4) Since the IUE fluxes do not clearly show the primary eclipse, it might be surmised that all stellar atmospheres are inappropriate. For instance, a black body at $T_{e} \simeq 22000 \mathrm{R}$ can represent most of the satellite spectrum satisfactorlly, but predicts too much flux for $\lambda<1200$. (5) Since the outburst cycle length is not incompatible with that for dwarf novae as a class, a thick, systemic disk may be considered to be associated with a thermal bremsstrahlung $\lambda^{-2}$ dependence. With a nominal unit Gaunt factor, $\mathrm{T} \simeq 3 \times 10^{5} \mathrm{~K}$ approximates part of the IUE continuum, but also predicts too much flux for $\lambda<1500$. Thus, it is Iikely that the continum is composite and varlable, as other spectra show, and a unique temperature assignment should not be expected.

Fig. 1 and 2 show the richness of the emission line spectra. Table I enumerates the fons and observed and rest wavelengths which have been tentatively identified.

TABLE I. Tentative Identifications for Emission Lines for V Sagittae

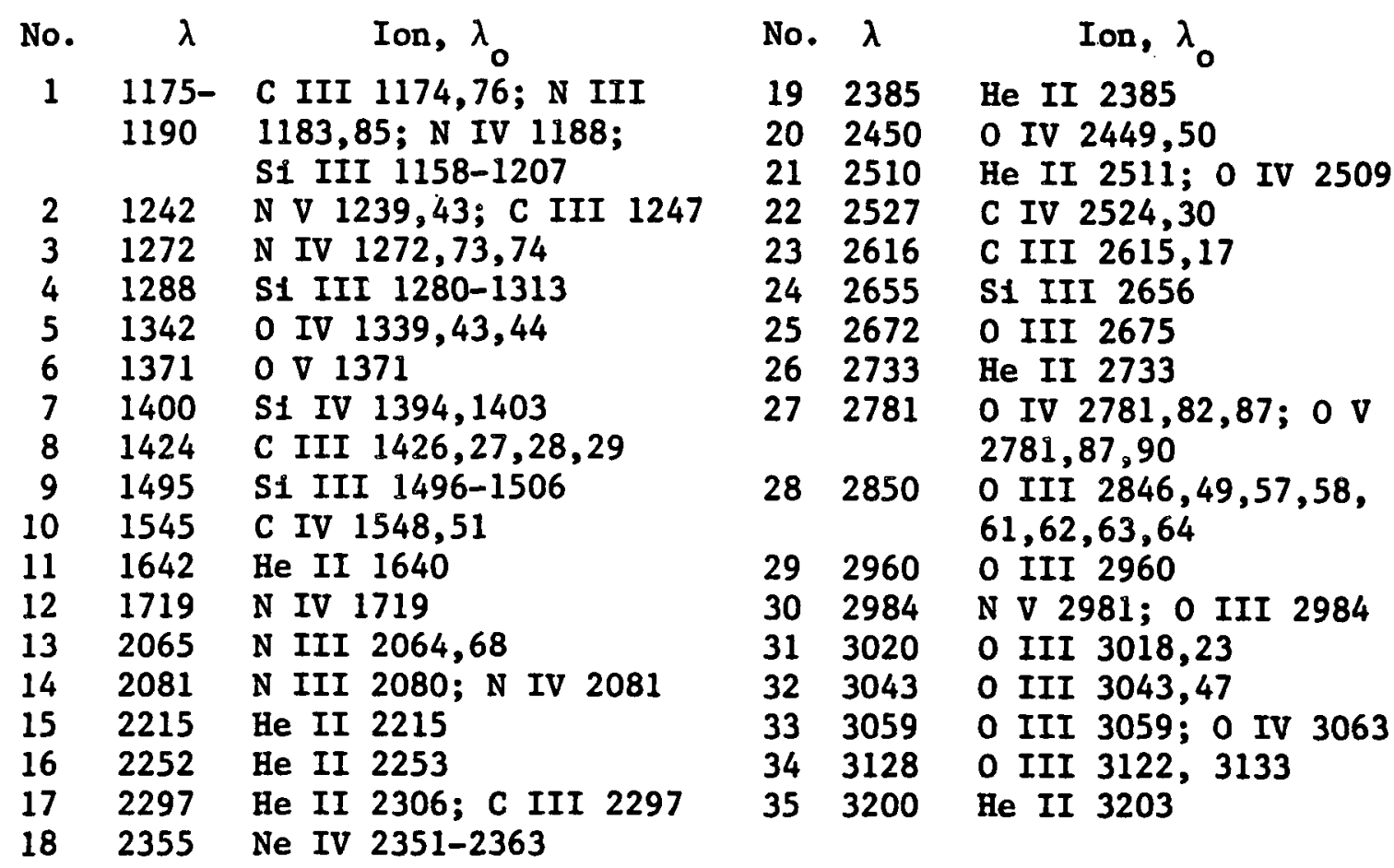

Due to cathode reseaux and to saturation effects for the longer exposures, not all lines are seen on all spectra. However, these effects alone cannot account for the intrinsic variablitity in strength of many emission features. Fig. 1 and 2, in fact, show that not all 1dentified Iines actually appear on these two samples even though they are convincingly present on most of the spectra. This behavior is consistent with the intrinsic variability noted by Herbig, et al. Additionally, 
there are 3 emissions seen on most spectra which cannot be identified at this time: $\lambda \lambda$ 1815, 2582, and 2930. For all these emission lines, there seem to be velocity variations, but these are probably spurious effects due to the variability in strength.

The ground-based spectra clearly show some emissions to be double and others likely to be single. The study of the IUE lines has been inhibited by the low spectral resolution which leaves unclear the possible duplicity of many of these lines and progress has been very slow. At the present, one may only conjecture that the several ionization stages could lead to the evaluation of temperature and density gradients in the line-forming volume.

An evolutionary interpretation for $V$ Sge continues to be obscure but some progress may eventually emerge if two assumptions are admitted: (a) the errors associated with the masses and radii given by Herbig, et al. are $\simeq 0.5 \odot$, and (b) the large object is the cool one. If these propositions are accepted, one can envision the large object to be an early $A$, near-main sequence star with perhaps $T_{e} \simeq 10^{4} \mathrm{~K}$. Interpreting the companion object only as a white dwarf is unsatisfactory, for the IUE spectra may then be affected by the A-type star, and this is not observed. A more likely possibility would consider a thick, hot disk embedding a white dwarf. A disk temperature, $17000 \mathrm{~K}<\mathrm{T}<3 \times 10^{5} \mathrm{~K}$, would be sufficiently high and the disk dimensions, limited by the Roche lobe, sufficiently large so that this structure dominated the IUE spectra. Interest in this configuration would rest largely in having a relatively hot, non-degenerate binary member rather than the familiar G- or K-type dwarf or subgiant. It could then be conjectured that $V$ Sge is now in a second stage of fast mass exchange and this could be consistent with the secular period shortening.

This work was supported by NASA grant NSG 5296, which is gratefully acknowledged.

\section{REFERENCES}

Campbe11, L. 1912, Ann. H.C.0. 63, 107.

$$
\text { 1918, ibid. } 79,69 \text {. }
$$

1938, Pop. Astr. 46, 112.

Carbon, D.F., and Gingerich,0. $\overline{1969}$, Proc. Third Harvard-Smithsonian Conference on Stellar Atmospheres 0. Gingerich, ed., M.I.T. Press, Cambridge, p. 377 .

FitzGerald, M.P. 1968, Astron.J. 73, 983.

Gaposchkin, S. 1952, Ann. H.C.0. 118, 53.

Herbig, G.H., Preston, G.W., Smak, J., and Paczynsk1, B. 1965, Astrophys. J. 141, 617.

Jacchla, L. 1931, Pub. Oss. Bologna 2, 89.

Jamar, C., Macau-Hercot, D., Monfils, A., Thompson, G.I., Houzlaux, L., and Wilson, R. 1976, Ultraviolet Bright Star Spectrophotometric Catalogue.

Robinson, E.L. 1976, Ann. Rev. Astron. \& Astrophys. 14, 119. Ryves, P.M. 1932, M.N.R.A.S., 92, 715. 